\title{
Association between serum neuron- specific enolase, age, overweight, and structural MRI patterns in 901 subjects
}

Johanna Hoffmann', Deborah Janowitz (1)', Sandra Van der Auwera ${ }^{1,2}$, Katharina Wittfeld², Matthias Nauck ${ }^{3,4}$, Nele Friedrich ${ }^{3,4}$, Mohamad Habes $\mathbb{1}^{1,5,6}$, Christos Davatzikos ${ }^{6}$, Jan Terock7, Martin Bahls ${ }^{4,8}$, Annemarie Goltz', Angela Kuhla ${ }^{9}$, Henry Völzke $e^{4,5,10}$ and Hans Jörgen Grabe ${ }^{1,2}$

\begin{abstract}
Serum neuron-specific enolase (SNSE) is considered a marker for neuronal damage, related to gray matter structures. Previous studies indicated its potential as marker for structural and functional damage in conditions with adverse effects to the brain like obesity and dementia. In the present study, we investigated the putative association between sNSE levels, body mass index (BMI), total gray matter volume (GMV), and magnetic resonance imaging-based indices of aging as well as Alzheimer's disease (AD)-like patterns. Subjects/Methods: sNSE was determined in 901 subjects (499 women, 22-81 years, BMI 18-48 kg/m²), participating in a population-based study (SHIP-TREND). We report agespecific patterns of sNSE levels between males and females. Females showed augmenting, males decreasing sNSE levels associated with age (males: $p=0.1052$, females: $p=0.0363$ ). sNSE levels and BMI were non-linearly associated, showing a parabolic association and decreasing SNSE levels at BMI values $>25(p=0.0056)$. In contrast to our hypotheses, sNSE levels were not associated with total GMV, aging, or AD-like patterns. Pathomechanisms discussed are: sex-specific hormonal differences, neuronal damage/differentiation, or impaired cerebral glucose metabolism. We assume a sex-dependence of age-related effects to the brain. Further, we propose in accordance to previous studies an actual neuronal damage in the early stages of obesity. However, with progression of overweight, we assume more profound effects of excess body fat to the brain.
\end{abstract}

\section{Introduction}

The global prevalence of obesity has more than doubled since 1980. In $2014,>1.9$ billion adults were overweight (body mass index (BMI) $\geq 25 \mathrm{~kg} / \mathrm{m}^{2}$ ), from which over 600 million were obese $\left(B M I \geq 30 \mathrm{~kg} / \mathrm{m}^{2}\right)^{1}$. The global world population is aging and gaining weight; therefore, we will face the problem of many overweight elderly people in the future. There is growing evidence that the consequences of excess body weight extend to the brain. Specifically,

Correspondence: Deborah Janowitz (janowitz@uni-greifswald.de)

${ }^{1}$ Department of Psychiatry and Psychotherapy, University Medicine Greifswald, Greifswald, Germany

${ }^{2}$ German Center for Neurodegenerative Diseases (DZNE), Site Rostock, Greifswald, Germany

Full list of author information is available at the end of the article

Johanna Hoffmann and Deborah Janowitz contributed equally to this work human $^{2}$ and animal studies ${ }^{3}$ reported effects of obesity on neuronal structure and function.

For example, obesity has been associated with increased gray matter (GM) damage ${ }^{4-6}$. However, the mechanism of how obesity, GM reductions, and cognitive alterations are related remains subject of ongoing discussion. Many studies reported midlife obesity to increase the risk for cognitive impairment ${ }^{2}$, and neurodegenerative diseases like Alzheimer's disease $(\mathrm{AD})^{7}$. Obesity accelerates neurodegenerative processes known in the aging brain $^{8}$, and initiates structural cerebral alterations that result in impairments of memory performance in aging ${ }^{5}$.

Recent studies established indices that acquire cerebral atrophy patterns. They allow to distinguish between agedependent atrophy (spatial pattern of atrophy for 
recognition of brain aging, SPARE-BA), and atrophy specifically found in clinically diagnosed AD cases (spatial patterns of abnormality for recognition of early $A D$, SPARE-AD) ${ }^{9}$ from magnetic resonance (MR) images. The SPARE-AD index is predictive for transition from normal cognition to mild cognitive impairment ${ }^{10}$, and further on to $\mathrm{AD}^{11}$. Habes et al. $^{9}$ demonstrated that waist circumference (WC) was associated with more advanced brain aging patterns in a male sub-cohort in our study population.

In order to give evidence for the presence and extent of cerebral damage and alterations, we need brain-specific markers. In this context, there is growing evidence, that the neuron-specific enolase (NSE) could be a good candidate. NSE is an enzyme of the glycolytic pathway and is closely related to the differentiated state of mature nerve cells ${ }^{12}$. In neurons, NSE is primarily localized in the cytoplasm. Since NSE cannot be secreted by cells, an increase of NSE in cerebrospinal fluid (CSF) or serum is a marker for neuronal damage $^{13}$. Using the Allen Brain Atlas, it was shown that NSE gene expression is increased in frontal and parietal lobes, claustrum, and cerebellum, with claustrum and cerebellum showing the highest gene expression ${ }^{14}$.

Previous studies demonstrated that different regions are affected by GM reduction in normal aging or AD. Cerebral alterations in aging or $\mathrm{AD}$ can be distinguished by SPARE-BA or SPARE-AD ${ }^{9}$, but no study investigated the association between aging patterns and serum neuronspecific enolase (sNSE) levels so far.

In addition of the effects of aging on GM, an increased body weight influences gray matter volume $(G M V)^{4}$. First evidence of a putative link between obesity-associated alterations in GMV and SNSE levels was established by the study of Mueller et al. ${ }^{14}$. The authors described an inverse correlation between SNSE and GM density in hippocampal and cerebellar regions in overweight young adults. Therefore, we sought to investigate if SNSE levels would be indicative of these alterations in GMV associated with obesity in our population-based sample.

Additionally, medication may interfere with measurements of cerebral volume and function. Therefore, we controlled calculations also for antihypertensive and lipidlowering drugs.

Based on findings in literature, we hypothesized, that

1. sNSE levels are positively associated with age,

2. SNSE levels are positively associated with increasing BMI or WC,

3. SNSE levels are negatively associated with GMV and structural aging patterns (SPARE-BA and SPARE-AD).

\section{Materials and methods Participants}

We collected data in course of the population-based Study of Health in Pomerania (SHIP) ${ }^{15-17}$. The study comprises adult residents living in three cities and 29 communities, with a total population of 212,157. SHIPTREND contains a stratified random sample of 8,016 adult Caucasians aged 20-79 years (baseline). Effectively, a total number of 4,420 subjects participated in the study. Baseline information was collected between 2008 and 2011. The only exclusion criterion for SHIP-TREND was participation in the parallel running study SHIP-0. The data of sNSE were collected within a subsample of SHIPTREND, consisting of 1,000 participants without clinical diabetes mellitus.

The study was approved by the ethics committee of the University of Greifswald adhering to the Declaration of Helsinki. All subjects provided written informed consent.

\section{Clinical examination and medication}

Clinical examination procedures have been described previously ${ }^{4}$.

Smoking was defined as current smoking (occasional; 1-14 cigarette(s) per day; $\geq 15$ cigarettes per day), former smoking (occasional; 1-14 cigarette(s) per day; $\geq 15$ cigarettes per day), and never smoking.

Current medication was recorded using Anatomical Therapeutic Chemical classification $\operatorname{codes}^{18}$. For our analysis, we used information on antihypertensive medication $\left(\mathrm{CO}^{*}, \mathrm{C}^{*}, \mathrm{C}^{*}, \mathrm{C}^{*}\right.$, and $\left.\mathrm{C} 09^{*}\right)$ and lipidlowering drugs $\left(\mathrm{C} 10^{*}\right)$.

\section{Laboratory analyses}

Fasting blood samples (fasting $\geq 8 \mathrm{~h}$ ) were drawn between before noon from the cubital vein of subjects in the supine position and analyzed immediately or stored at $-80^{\circ} \mathrm{C}$. Serum concentrations of sNSE were determined using an immunoassay (cobas e 411 analyzer, Roche Diagnostics GmbH, Mannheim, Germany) with a functional sensitivity of $0.05 \mu \mathrm{g} / \mathrm{L}$. The interassay variation was $4.4 \%$.

Photometry was used to quantify high-density lipoprotein cholesterol (HDL-C) concentrations (Hitachi 704, Roche Diagnostics). Comparability in the longitudinal HDL-C analyses was ensured by using baseline HDL-C concentrations as the reference, and calculating corrected follow-up HDL-C concentrations based on a previous published conversion formula (HDL_fu_corr $=-80+$ $(1.158 \times$ HDL_fu $))^{19}$. We quantified serum low-density lipoprotein (LDL-C) by applying a precipitation procedure using dextran sulphate (Immuno, Heidelberg, Germany) on an Epos 5060 (Eppendorf, Hamburg, Germany). LDLC, HDL-C, and total cholesterol were measured in $\mathrm{mmol} /$ $\mathrm{L}$ as dimensional scores.

The laboratories analysing the samples of SHIP, participate in the official German external quality proficiency testing programmes. As often as available, all assays were calibrated against the international reference preparations. 


\section{Magnetic resonance imaging}

We asked participants to undergo whole-body magnetic resonance imaging (MRI). The image acquisition parameters of the whole-body MRI scans in SHIP have been described previously ${ }^{20}$. The images were acquired with 1.5 Tesla scanner (Magnetom Avanto: Siemens Medical Solutions, Erlangen, Germany). MRI images were available for all 901 subjects with information on SNSE. Subjects who fulfilled exclusion criteria against MRI (e.g., pregnancy, cardiac pacemaker), who refused participation and subjects with a stroke, Parkinson's disease, epilepsy, hydrocephalus, enlarged ventricles, pathological lesions, history of cerebral tumor, and multiple sclerosis were excluded from the present analyses. Moreover, we excluded images with severe inhomogeneities of the magnetic field, strong movement artefacts, and images, who failed for quality control. Within the voxel based morphometry (VBM), 8 toolbox homogeneity check was conducted. After exclusion, 832 subjects remained in the sample (Supplementary Fig. 4). For detailed information for exclusion criteria of MRI of the brain in SHIPTREND, see Supplementary Fig. 5.

For structural examination, the three-dimensional T1weighted axial MRI sequence with the following parameters was used: $1.900 \mathrm{~ms}$ repetition time, $3.4 \mathrm{~ms}$ echo time, flip angle $=15$, and a voxel size of $1.0 \times 1.0 \times 1.0$ $\mathrm{mm}$. GM, white matter, and the volume of the CSF were determined using SPM 8 with the VBM toolbox for spatially normalization by the means of high-dimensional DARTEL, bias correction, and segmentation into the different tissue classes of the T1 images. Intracranial volume (ICV) was calculated as the sum of GM, white matter, and CSF.

\section{MRI pattern classification}

The SPARE-AD is an index previously developed using a support vector machine classifier that allows distinguishing between atrophy patterns in regions typically affected by clinical AD. It predicts the transformation from normal cognition to mild cognitive impairment and further on to clinical AD. Furthermore, we included in this study the SPARE-BA index to capture brain aging patterns of atrophy. The method of SPARE-BA has been described in more details earlier'.

\section{Statistical analysis}

In 99 subjects, sNSE was not measurable, thus data about sNSE levels were available for $N=901$ participants. To detect sex-dependent differences on a descriptive level, we divided the sample in females and males. To detect BMI-dependent differences on a descriptive level, we divided the sample in two groups. The overweight group contained participants with a BMI $\geq 25 \mathrm{~kg} / \mathrm{m}^{2}$, the normalweighted group subjects with a BMI $<25 \mathrm{~kg} / \mathrm{m}^{2}$. The $\chi^{2}$ test was used to evaluate differences between groups for categorical variables (e.g., sex or medication) and the independent samples $t$ test to compare means of continuous data (e.g., age or sNSE concentrations). Associations between sNSE levels and age, sex, BMI, vascular risk factors, or GMV were performed using linear regression analyses with robust estimates with STATA/MP version 13.1 (StataCorp, TX 77845, USA). Bootstrap analyses with 1,000 replicates were used to evaluate the robustness of the models. This did not change the results of calculations of the association between SNSE and age, specifically the sex-separated analyses $(p=0.008)$, nor the results of calculations of a non-linear association between SNSE, BMI, and WC (BMI $p=0.0056$, WC $p=0.0049$ ). In calculations concerning an association between SNSE and BMI, the non-linear gave a better model fit $\left(R^{2}\right.$ increase from 2.2 to $2.9 \%$ ). Thus, we included cubic splines for BMI. Statistical significance was defined as $p<0.05$. Hypertension was defined by a systolic blood pressure (BP) $\geq 140 \mathrm{Hg}$ and/or a diastolic BP $\geq 90$ and/or antihypertensive intake. We further tested for associations between sNSE levels and SPARE-BA or SPARE-AD. Linear regression analyses with robust estimates on the dependent variable sNSE or GMV were performed. The analyses were adjusted for age and sex as basic confounders. As we observed an interaction term between age and sex, we included this interaction term in all further regression models. Analyses for GM were additionally adjusted for ICV and analyses for blood lipids and blood pressure were additionally adjusted for medication. Tests for non-linearity were performed graphically using lowess-smoothing plots for the full sample as well as sexseparated to assess possible interaction effects with sex. Analyses for blood pressure, hypertension, and blood lipids were controlled for medication. Specifically, all calculations were adjusted for age, sex, and age $\times$ sex interaction. Calculations on triglycerides, LDL-C, HDL-C, total cholesterol were additionally adjusted for lipidlowering drugs. Calculations on systolic and diastolic blood pressure were additionally adjusted for antihypertensives.

\section{Results}

\section{Sample characteristics}

In total, we included 901 subjects (499 (55.4\%) female) for the main analysis (Supplementary Table 1).

Table 1 shows the descriptive statistics of sexdependent differences in the sample. Compared with males, females had lower GMV, BMI, WC, systolic and diastolic BP, hypertension, and triglycerides, but higher HDL-C and total cholesterol on a descriptive level. The difference between males and females regarding smoking nicotine was also significant: Females were more often current smokers, whereas males were more often former 
Table 1 Descriptive sample characteristics: group comparison of females and males

\begin{tabular}{|c|c|c|c|c|c|}
\hline & $\begin{array}{l}\text { Females } \\
\mathrm{N} / \text { mean }\end{array}$ & $\pm S D$ & $\begin{array}{l}\text { Males } \\
\text { N/mean }\end{array}$ & $\pm \mathrm{SD}$ & $p$ value \\
\hline Participants & 499 & & 402 & & \\
\hline Age $(y)$ & 50.45 & \pm 13.09 & 50.16 & \pm 14.16 & 0.7449 \\
\hline NSE ( $\mu \mathrm{g} / \mathrm{L})$ & 8.73 & \pm 3.81 & 8.95 & \pm 3.57 & 0.3739 \\
\hline $\mathrm{GMV}\left(\mathrm{cm}^{3}\right)^{*}$ & 557.77 & \pm 55.66 & 606.1 & \pm 65.68 & $<0.001$ \\
\hline BMI $\left(\mathrm{kg} / \mathrm{m}^{2}\right)$ & 26.83 & \pm 4.86 & 27.7 & \pm 3.68 & 0.0029 \\
\hline WC (cm) & 82.85 & \pm 11.71 & 94.09 & \pm 10.95 & $<0.001$ \\
\hline $\begin{array}{l}W C \geq 88(\mathrm{~cm}) \\
\text { women } / \geq 102(\mathrm{~cm}) \\
\text { men }\end{array}$ & 379 & & 315 & & 0.027 \\
\hline Systolic BP in mmHg & 118.83 & \pm 15.59 & 131.2 & \pm 15.25 & $<0.001$ \\
\hline $\begin{array}{l}\text { Diastolic BP in } \\
\mathrm{mmHg}\end{array}$ & 74.35 & \pm 15.59 & 79.21 & \pm 9.89 & $<0.001$ \\
\hline Hypertension & 178 & & 179 & & $<0.001$ \\
\hline Smoking nicotine & & & & & $<0.001$ \\
\hline Current & 254 & & 130 & & \\
\hline Former & 141 & & 184 & & \\
\hline \multicolumn{6}{|l|}{ Never } \\
\hline $\begin{array}{l}\text { Triglycerides in } \\
\mathrm{mmol} / \mathrm{L}\end{array}$ & 1.28 & \pm 0.67 & 1.58 & \pm 1.03 & $<0.001$ \\
\hline LDL-C in $\mathrm{mmol} / \mathrm{L}$ & 3.4 & \pm 0.9 & 3.44 & \pm 0.88 & 0.5386 \\
\hline $\mathrm{HDL}-\mathrm{C}$ in $\mathrm{mmol} / \mathrm{L}$ & 1.61 & \pm 0.36 & 1.32 & \pm 0.3 & $<0.001$ \\
\hline $\begin{array}{l}\text { Total cholesterol in } \\
\mathrm{mmol} / \mathrm{L}\end{array}$ & 5.59 & \pm 1.04 & 5.37 & \pm 1.03 & 0.0022 \\
\hline Antihypertensives & 153 & & 109 & & 0.137 \\
\hline Lipid-lowering drugs & 34 & & 36 & & 0.143 \\
\hline
\end{tabular}

$B M I$ body mass index, $B P$ blood pressure, GMV gray matter volume, $H D L-C$ highdensity lipoprotein cholesterol, LDL-C low-density lipoprotein cholesterol, NSE neuron-specific enolase, WC waist circumference, $y$ years

*Data available for 832 subjects; bold values represent statistical significant $p$ values defined as $p<0.05$

smokers. No differences were seen for age, NSE, LDL-C, antihypertensive and lipid-lowering drugs in the group comparisons. Table 2 shows the descriptive analysis of BMI-dependent differences in the sample. Men were more often overweight than women were, and overweight subjects were older compared to individuals with normal weight $(p<0.001)$. Figure 1 shows a scatter plot with the linear increase of BMI during aging. GMV was lower in the overweight group $(p=0.004)$ in a subcohort of 832 subjects with MRI assessment. Obesity-associated comorbidities as hypertension and increased blood lipids were more often in the overweight group $(p<0.001)$, as well as antihypertensive intake $(p<0.001)$. Apart from
Table 2 Descriptive sample characteristics: group comparisons in terms of the BMI

\begin{tabular}{|c|c|c|c|c|c|}
\hline & $\begin{array}{l}\text { BMI } \\
<25 \mathrm{~N} / \\
\text { mean }\end{array}$ & $\begin{array}{l} \pm \mathrm{SD} \\
\text { or (\%) }\end{array}$ & $\begin{array}{l}\text { BMI } \\
\geq 25 \mathrm{~N} / \\
\text { mean }\end{array}$ & $\begin{array}{l} \pm \text { SD or } \\
(\%)\end{array}$ & $p$ value \\
\hline Participants & 302 & (33.5) & 599 & $(66.5)$ & \\
\hline Sex & & & & & $<0.001$ \\
\hline Women & 203 & $(40.7)$ & 296 & (59.3) & \\
\hline Men & 99 & $(24.6)$ & 303 & $(75.4)$ & \\
\hline Age $(y)$ & 44.95 & \pm 13.4 & 53.0 & \pm 12.8 & $<0.001$ \\
\hline NSE $(\mu \mathrm{g} / \mathrm{L})$ & 9.0 & \pm 3.6 & 8.8 & \pm 3.7 & 0.407 \\
\hline GMV $\left(\mathrm{cm}^{3}\right)^{*}$ & 590.4 & \pm 66.5 & 576.8 & \pm 63.2 & 0.004 \\
\hline WC (cm) & 76.2 & \pm 7.2 & 93.7 & \pm 10.6 & $<0.001$ \\
\hline $\begin{array}{l}\text { WC } \geq 88(\mathrm{~cm}) \\
\text { women }\end{array}$ & 1 & $(0.7)$ & 147 & (99.3) & $<0.001$ \\
\hline$W C \geq 102(\mathrm{~cm})$, men & 0 & $(0.0)$ & 104 & $(100.0)$ & $<0.001$ \\
\hline Systolic BP in mmHg & 116.4 & \pm 13.9 & 128.4 & \pm 16.4 & $<0.001$ \\
\hline Diastolic BP in mmHg & 72.4 & \pm 8.2 & 78.6 & \pm 9.6 & $<0.001$ \\
\hline Hypertension & 45 & $(12.6)$ & 312 & $(87.4)$ & $<0.001$ \\
\hline Smoking nicotine & & & & & 0.013 \\
\hline Current & 72 & $(23.8)$ & 118 & (19.7) & \\
\hline Former & 89 & $(29.5)$ & 236 & (39.4) & \\
\hline Never & 140 & $(46.4)$ & 244 & $(40.7)$ & \\
\hline $\begin{array}{l}\text { Triglycerides in } \\
\mathrm{mmol} / \mathrm{L}\end{array}$ & 1.0 & \pm 0.4 & 1.6 & \pm 1.0 & $<0.001$ \\
\hline LDL-C in $\mathrm{mmol} / \mathrm{L}$ & 3.1 & \pm 0.9 & 3.5 & \pm 0.9 & $<0.001$ \\
\hline $\mathrm{HDL}-\mathrm{C}$ in $\mathrm{mmol} / \mathrm{L}$ & 1.6 & \pm 0.4 & 1.4 & \pm 0.3 & $<0.001$ \\
\hline $\begin{array}{l}\text { Total cholesterol in } \\
\mathrm{mmol} / \mathrm{L}\end{array}$ & 5.3 & \pm 1.0 & 5.6 & \pm 1.0 & $<0.001$ \\
\hline Antihypertensives & 33 & $(12.6)$ & 229 & $(87.4)$ & $<0.001$ \\
\hline Lipid-lowering drugs & 6 & (8.6) & 64 & $(91.4)$ & $<0.001$ \\
\hline
\end{tabular}

$B M I$ body mass index, $B P$ blood pressure, GMV gray matter volume, $H D L-C$ highdensity lipoprotein cholesterol, $L D L-C$ low-density lipoprotein cholesterol, NSE neuron-specific enolase, WC waist circumference, $y$ years

*Data available for 832 subjects; bold values represent statistical significant $p$ values defined as $p<0.05$

HDL-C, all blood lipids (triglycerides, LDL-C, and total cholesterol) and lipid-lowering drug intake were higher in the overweight group $(p<0.001)$. Former smokers were more often in the obese group. Overweight showed somewhat lower sNSE levels than normal weighted, but the difference was not significant.

\section{Association between sNSE with age}

We did not find linear association between age and sNSE levels $(p=0.205$, beta $=0.0122)$ (Table 3$)$. As we observed different age effects in males and females, we 


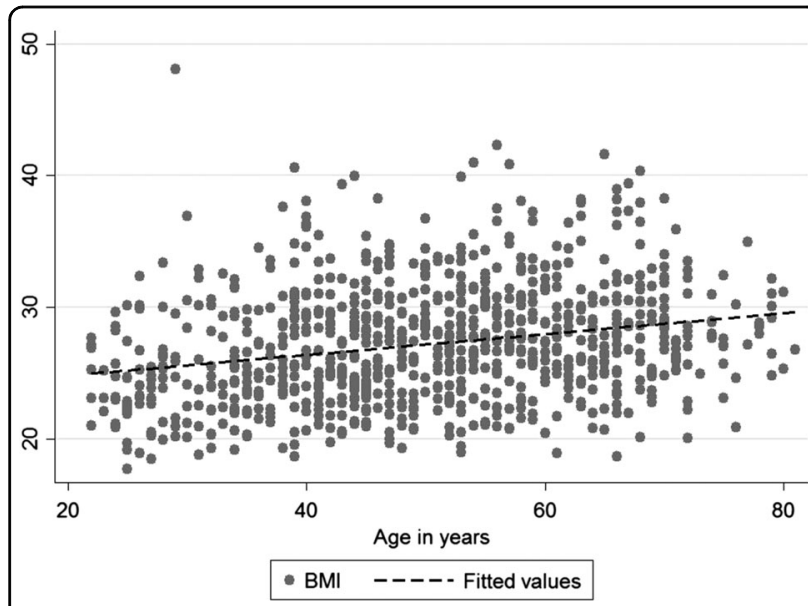

Fig. 1 Increasing patterns of BMI during aging. Subjects show an increasing BMI with advancing age. Linear trend line, no adjustment. $\mathrm{BMl}$ in $\mathrm{kg} / \mathrm{m}^{2}$

Table 3 Association of sNSE levels with age (non-linear), sex, and sex $\times$ age interaction

\begin{tabular}{lllll}
\hline & Beta & SE & $\boldsymbol{T}$ & $\boldsymbol{p}$ value \\
\hline Age $(\mathrm{y})^{* * *}$ & & & $F=1.05$ & 0.37 \\
Sex* & -0.221 & 0.2467 & -0.9 & 0.371 \\
Age $\times$ sex interaction & 0.05 & 0.019 & 2.64 & 0.009 \\
\hline
\end{tabular}

SNSE serum neuron-specific enolase

*Unadjusted

\#Variables were treated non-linear as splines; no betas, SE possible

included sex-stratified cubic splines for age (four knots equally placed; males: $26,44,57,72$ and females: 28,45 , $56,71)$ and included an age $\times$ sex interaction term. We observed a significant age-sex interaction $(p=0.009)$. The levels of sNSE in aging are depicted as scatter plots separately for males and females in Supplementary Fig. 1. In sex-specific analyses, the association between age and sNSE was not significant $(p=0.1052)$ for males, whereas females showed a significant association between age and sNSE levels $(p=0.0363)$. For males, a U-shaped and for females a J-shaped relation between age and SNSE was observed (Fig. 2).

\section{Association between sNSE and BMI}

sNSE levels decreased with increasing BMI in the linear model (Supplementary Fig. 2). In the non-linear model, sNSE values and BMI showed a U-shaped negative association (Supplementary Fig. 3). These effects were significant for both, BMI $(p=0.0056)$ and WC $(p=0.0042)$, apart from an influence of age and sex. Specifically, sNSE levels increased with higher BMI values up to a BMI of 25 $\mathrm{kg} / \mathrm{m}^{2}$ and decreased thereafter (Fig. 3). $p$ values for the

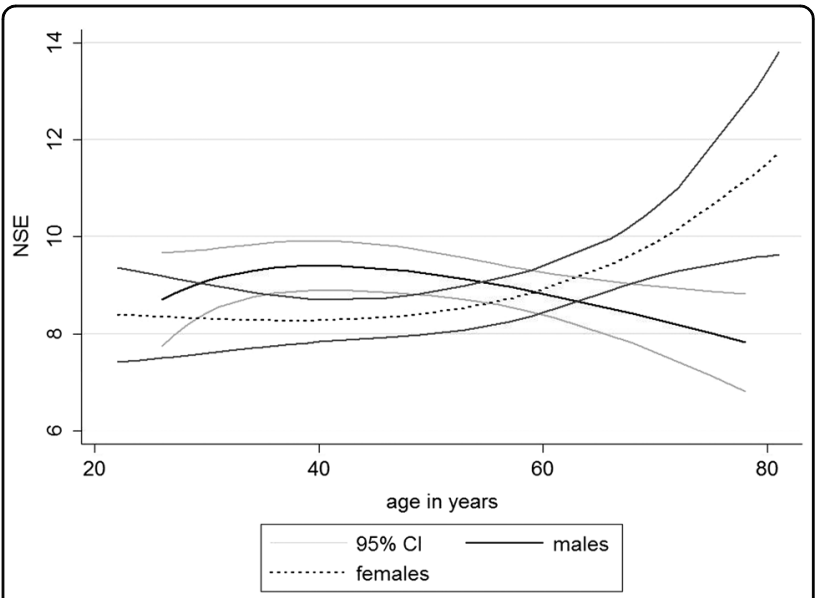

Fig. 2 Non-linear age-dependent sNSE levels in men and women. Women show constant low sNSE levels in younger years and increasing levels in higher ages, whereas men show increasing SNSE levels in young adulthood and a decrease in older ages. About an age of 60 years, sNSE values are similar in men and women. Sex-specific differences in the hormonal balance may serve as explanatory approach. After menopause, women have both lower levels of estradiol and testosterone. The missing estradiol-mediated neuroprotective effects may result in increased neural death, showing in increased sNSE levels. sNSE in $\mu \mathrm{g} / \mathrm{L}$

association between sNSE levels and BMI or WC were similar; therefore, we performed the following analyses with BMI only. To investigate presumed age-dependent differences in overweight-associated cerebral impairment, we stratified the sample for age. Regarding a possible interaction between BMI and age, the interaction term was not significant $(p=0.244)$, but when stratifying the sample into different age groups (age $<40$, age 40-60, age $>60$ ), only the oldest sample revealed a significant association between SNSE and BMI $(p=0.02)$. When regarding the overweight and normal-weighted separately, a significant association between SNSE levels and BMI was specific for overweight elderly $(p=0.0365)$. Concerning hypertension, blood pressure, blood lipids and smoking status, only HDL $(p=0.016)$, and current smoking ( $p=$ 0.019 ) exhibited a significant inverse association with sNSE in the full sample (Table 4).

\section{Association between SNSE and GMV, SPARE-BA and SPARE-AD}

sNSE levels were not associated with GMV $(p=0.963)$ after adjustment for ICV (Table 5). Stratifying the sample into subjects with and without overweight parallel to Mueller et al., results were also not significant (BMI $<25$ : $p=0.854 ; \mathrm{BMI} \geq 25: p=0.416)$. Overweight elderly aged $>60$ years, showed a negative association with GMV, yet the association was not significant. In normal-weighted elderly, this tendency was not seen (BMI $<25: p=0.65$; $\mathrm{BMI} \geq 25: p=0.068$ ). Also no significant associations with 


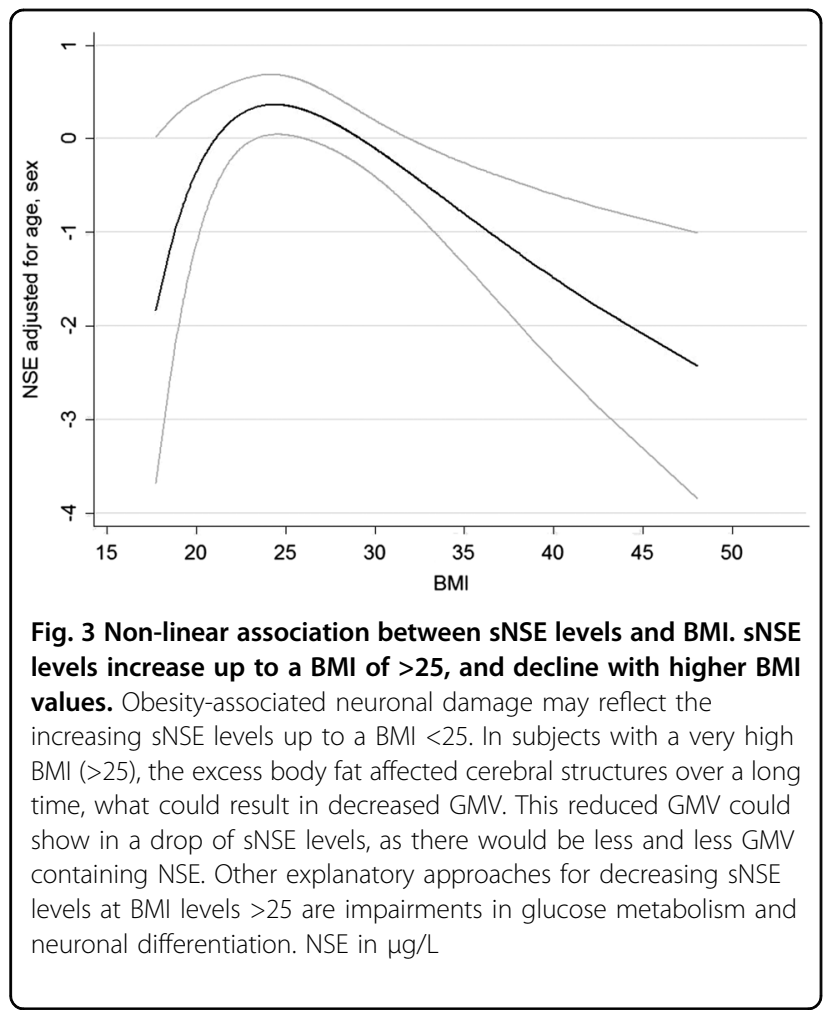

SNSE and SPARE-BA $(p=0.928)$ or SPARE-AD $(p=$ 0.643 ) were observed in the total sample or in elderly aged $>65$ years.

However, SPARE-BA and SPARE-AD were associated with GMV $(p<0.001)$.

\section{Discussion}

We showed sex-dependent differences in the patterns of sNSE levels with increasing age, with an increase of sNSE levels in elderly women.

sNSE levels and BMI were non-linearly associated, showing a parabolic association with decreasing sNSE levels at BMI values $>25 \mathrm{~kg} / \mathrm{m}^{2}$.

We could not substantiate the hypothesis of an inverse association between SNSE levels and GMV, yet we saw the tendency in elderly subjects with overweight. Atrophy patterns in brain aging (SPARE-BA) or for AD-like patterns of atrophy (SPARE-AD) were not associated with sNSE levels.

\section{Association between SNSE and age}

The effect of age on NSE concentrations is differently discussed in the literature, partly because NSE levels may be assessed in CFS or in serum ${ }^{13,21,22}$. Until now, only NSE levels in CSF and age were positively associated ${ }^{22}$. In contrast, serum NSE levels showed no age-dependent alterations in two studies with $N=108$ and $N=41$ probands $^{13,21}$. Corresponding to previous studies based on
Table 4 Associations of sNSE levels with BMI, WC, and lipids

\begin{tabular}{lllll}
\hline & Beta & SE & $\boldsymbol{T}$ & $\boldsymbol{p}$ value \\
\hline BMI $\left(\mathrm{kg} / \mathrm{m}^{2}\right)^{\#}$ & & & $F=4.22$ & $\mathbf{0 . 0 0 5 6}$ \\
WC $(\mathrm{cm})^{\#}$ & & & $F=4.44$ & $\mathbf{0 . 0 0 4 2}$ \\
Triglycerides in mmol/L* & -0.19 & 0.1379 & -1.38 & 0.168 \\
LDL-C in mmol/L* & 0.1076 & 0.152 & 0.71 & 0.479 \\
HDL-C in mmol/L* & 0.8991 & 0.3734 & 2.41 & $\mathbf{0 . 0 1 6}$ \\
Total cholesterol in mmol/L* & 0.1611 & 0.1369 & 1.18 & 0.24 \\
Lipid-lowering drugs $^{1}$ & -0.0684 & 0.4902 & -0.14 & 0.889 \\
Smoking nicotine & & & & \\
Current (reference) & & & & \\
Former & -0.2799 & 0.2903 & -0.96 & 0.335 \\
Never & -0.7749 & 0.3309 & -2.34 & $\mathbf{0 . 0 1 9}$ \\
\hline
\end{tabular}

$B M I$ body mass index, HDL-C high-density lipoprotein cholesterol, LDL-C lowdensity lipoprotein cholesterol, sNSE serum neuron-specific enolase, WC waist circumference

Associations adjusted for age (non-linear), sex, and age $\times$ sex interaction "Variables were treated non-linear as splines; no betas, SE possible

*Additionally adjusted for lipid-lowering drugs; bold values represent statistical significant $p$-values defined as $p<0.05$

Table 5 Association of sNSE levels with GMV and SPAREBA and SPARE-AD

\begin{tabular}{lllll}
\hline & Beta & SE & $\boldsymbol{T}$ & $\boldsymbol{p}$ value \\
\hline GMV* $^{*}$ & -0.0116 & 0.2462 & -0.05 & 0.963 \\
SPARE-BA & -0.0001 & 0.0106 & -0.09 & 0.928 \\
SPARE-AD & 0.0045 & 0.0097 & 0.46 & 0.643 \\
\hline
\end{tabular}

GMV gray matter volume, sNSE serum neuron-specific enolase, SPARE-AD spatial patterns of abnormality for recognition of early Alzheimer's disease, SPARE-BA spatial pattern of atrophy for recognition of brain aging, WC waist circumference Associations adjusted for age (non-linear), sex, and age $\times$ sex interaction GMV, SPARE-AD/BA treated as outcome

*Additionally adjusted for ICV, data available for 832 subjects

SNSE levels ${ }^{13}$, 21 , we report no significant linear association between NSE and age. Considering the extensive neural loss in the course of $\mathrm{AD}$, studies have aimed to show disease-associated alterations in sNSE levels. However, studies produced inconsistent results concerning sNSE levels in $\mathrm{AD}^{23,24}$. Chaves et $\mathrm{al}^{23}$ found no differences in SNSE levels between AD patients and healthy elderly controls.

However, we did find significant non-linear sex-specific associations with aging. Specifically, females showing augmented, males decreasing sNSE levels associated with aging. We saw no association between sex and sNSE levels, which is in line with previous studies ${ }^{13}$, but an interaction between sex and age on SNSE levels. It seems contradictive that there was no association between sex and sNSE levels, but an association between SNSE levels 
and sex depending on age. Specific differences between the sNSE levels of men and women may only show with advancing age. Therefore, no association would be found when regarding participants of all ages, for the differences between males and females would be leveled.

To our knowledge, our study is the first to describe a divergence of age-dependent sNSE levels in women and men in a large sample from the general population $(N=$ 901). One may speculate sex-specific differences in the hormonal balance may serve as an explanatory approach. However, currently no research has further explored this association. Levels of sex steroids as estradiol and testosterone decline with age in both sexes. After menopause, estradiol levels show sharp descends in women, whereas testosterone levels decline more gradually in men. Elderly women have both lower levels of estradiol and testosterone ${ }^{25}$, accompanied by higher levels of cholesterol. This may be explained by increasing NSE levels, as enolases mobilize cholesterol ${ }^{26}$.

There is growing evidence for estradiol mediating neuroprotective effects in the hippocampus ${ }^{27}$. Since NSE is related to hippocampal structures, a possible explanation for our results is that the decline of estradiolmediated neuroprotective effects is accompanied by accelerated neuronal death eventually resulting in increased sNSE levels.

\section{Association between sNSE and BMI}

Our hypotheses of a positive association between SNSE levels and BMI was based on the study of Mueller et $\mathrm{al}^{14}$. They described an inverse correlation between GM density in cerebellum and hippocampus and sNSE levels in $N=27$ overweight subjects. They hypothesized that increased sNSE levels are a result of obesity-associated structural damage of GM. Accordingly, obesity-associated neuronal damage could reflect our findings of increasing sNSE levels up to a BMI $<25$. Moreover, the trend towards higher sNSE levels in overweight subjects in our study confirms the findings of Mueller et al. They described sNSE levels to be in the reference range in all subjects, but the overweight showed levels near the upper limit $(18.3 \mu \mathrm{g} / \mathrm{L})^{14}$.

We report a parabolic association between sNSE levels and BMI, with decreasing sNSE levels at BMI values $>25$. Moreover, in higher ages only overweight subjects showed a negative association with SNSE levels. Yet, we could not see the tendency in young adults, as depicted in the sample of Mueller et al. This leads to the assumption, that there may be age-dependent differences concerning an association between sNSE levels in overweight subjects.

\section{Association between sNSE and GMV, SPARE-BA and SPARE-AD}

We did not see a linear association between GMV or SPARE-BA or SPARE-AD sNSE levels in our sample. As a marker for neuronal injury, elevated sNSE levels result from an actual cerebral destruction, especially from GM damage. sNSE levels have shown to correlate positively with the severity level of the head injury, and therefore with the extent of brain cell damage ${ }^{28}$. It could be assumed, in subjects with a very high BMI, the excess body fat affected cerebral structures over a long time, resulting in decreased GMV. This reduced GMV could show in a drop of sNSE levels, as there would be less and less GMV containing NSE. Decreased sNSE levels have been reported in higher extent of brain atrophy in $\mathrm{AD}^{23}$, accordingly similar dynamics of sNSE levels can be expected in an obesity-related negative association with GMV.

The sample of Mueller et al. contained young adults (average age in the overweight group $26.4 \pm 5.4$ years), whereas in our sample the average age was in middle adulthood (average age in the overweight group 53.0 \pm 12.8). Thus, the detrimental effects of body fat could not have affected the brain for a long time. Further, we have seen a non-significant association between SNSE levels and GMV in obese elderly, but not in non-obese subjects, which is also in agreement with the findings of Mueller et $\mathrm{al}^{14}$. The study of Mueller et al. ${ }^{14}$ stated, that the higher the BMI, the greater the loss of GM density, comparable with mild cognitive impairment in the elderly. An induction of progressive brain alterations in obese subjects might be discussed, as studies revealed obesityassociated increased oxidative stress $^{29}$, and chronic inflammation ${ }^{30}$.

Furthermore, decreased sNSE levels could result from alterations in the glucose metabolism: As glycolytic enzyme, NSE is directly involved in the metabolism of glucose and obesity-related impairment in glucose metabolism in the frontal cortex have been reported ${ }^{31}$.

Another explanatory approach is neuronal differentiation. NSE is a specific marker for mature nerve cells, and is closely correlated to the differentiated state ${ }^{12}$. Depending on tissue and development state, different combinations of the subunits are expressed. $\gamma \gamma$ is specific for mature neurons with full synaptic connections, whereas during neuronal migration, hybrid enolases $(\alpha \gamma)$ are found (non-neuronal enolase) ${ }^{32}$. Obesity-related impairments on hippocampal neurogenesis have been demonstrated in animal studies with mice receiving a high-fat diet ${ }^{33,34}$. As NSE is associated with hippocampal structures, it can be speculated that obesity-related alterations of neuronal maturation and neurogenesis extend to the development of NSE.

In conclusion, we propose in accordance to previous studies an actual neuronal damage in the early stages of obesity. However, with progression of overweight, we assume more profound effects of excess body fat to the brain. 
Due to the cross-sectional design, we cannot investigate if the alterations in SNSE levels precede the onset of obesity or are resulting from the adverse effects of an enhanced bodyweight. As one study showed that a high BMI in mid-life was associated with temporal atrophy 24 years later ${ }^{35}$ and NSE is an indicator for neuronal injury, the current data support the second hypothesis.

\section{Strength and limitations}

Strength of this study is the cross-sectional populationbased sample. Thus, we can assume a sample, which is representative for the population. Stratification by age adds valuable information specific for particular age groups. With consideration of pattern classifiers in brain atrophy, we are able to make statements concerning the impact of average or increased aging processes on SNSE levels.

We measured height and weight by research assistants, therefore, we can rule out false statements concerning the BMI. As we performed analyses with BMI and WC, we can preclude effects that are unique for one measurement of obesity. We did not include data for deficits in glucose metabolism, thus this consideration should be examined in further research. To prevent confounding, we controlled for medication. However, we cannot rule out other confounding factors that may have affected the calculations.

\section{Conclusions}

Our study presents an analysis of a well-described cohort with a large number of participants, providing valuable and novel insights into alterations of sNSE levels in aging and obesity. We were able to show that sNSE levels and obesity are nonlinearly associated, even after adjusting for important confounders. Moreover, only in women, age was associated with increasing sNSE levels, thus we assume sex-specific pathomechanisms. With the determination of NSE levels in serum, we provide a valuable, easy determinable marker to get insights into brain-specific cellular alterations. Future studies, focusing on cerebral manifestations of obesity and the effect of their therapy would help to get to better and more individualized therapy strategies and to prevent or even undo cognitive impairment.

\footnotetext{
Acknowledgements

The SHIP is supported by the German Federal Ministry of Education and Research (grants 01ZZ9603, 01ZZ0103, and 01ZZ0403) and the German Research Foundation (DFG; GR 1912/5-1). MRI scans were supported by the Federal Ministry of Education and Research (grant 03ZIK012) and a joint grant from Siemens Healthineers, Erlangen, Germany, and the Federal State of Mecklenburg-West Pomerania. This cohort is part of the Community Medicine Research net (CMR) of the University of Greifswald, which is funded by the German Federal Ministry of Education and Research and the German Ministry of Cultural Affairs, as well as by the Social Ministry of the Federal State of Mecklenburg-West Pomerania. CMR encompasses several research projects that share data from the population-based SHIP. The work is also supported by
}

the German Research Foundation (DFG; GR 1912/5-1) and the Greifswald Approach to Individualized Medicine (GANI_MED) network funded by the Federal Ministry of Education and Research (grant 03IS2061A). We thank all staff members and participants of the SHIP studies. Measurement of NSE levels were financed by the Research Network Community Medicine.

\section{Authors' contributions}

All authors were involved in review and final approval of the manuscript. Specifically, M.N., N.F., M.H., C.D., J.H., D.J. and H.V. were involved in data collection, study design, and development of methods. S.V.d.A., J.H., D.J. and K.W. were involved in data analysis. M.B., H.J.G., A.G., A.K., and J.T. critically revised the manuscript for important intellectual content. J.H. and D.J. were responsible for writing the manuscript.

\section{Author details}

'Department of Psychiatry and Psychotherapy, University Medicine Greifswald, Greifswald, Germany. ${ }^{2}$ German Center for Neurodegenerative Diseases (DZNE), Site Rostock, Greifswald, Germany. Institute of Clinical Chemistry and Laboratory Medicine, University Medicine Greifswald, Greifswald, Germany. ${ }^{4}$ DZHK (German Centre for Cardiovascular Research), Partner site Greifswald, Greifswald, Germany. ${ }^{5}$ Institute for Community Medicine, University Medicine Greifswald, Greifswald, Germany. ${ }^{6}$ Department of Radiology, Section of Biomedical Image Analysis, University of Pennsylvania, Philadelphia, PA, USA. ${ }^{7}$ Department of Psychiatry and Psychotherapy, University Medicine Greifswald, HELIOS Hospital Stralsund, Stralsund, Germany. ${ }^{8}$ Department of Internal Medicine B, University Medicine Greifswald, Greifswald, Germany. ${ }^{9}$ Institute for Experimental Surgery, Rostock University Medical Center, Rostock, Germany. ${ }^{10}$ DZD (German Centre for Diabetes Research), Site Greifswald, Greifswald, Germany

\section{Competing interests}

The authors declare that they have no competing financial interests.

Publisher's note: Springer Nature remains neutral with regard to jurisdictional claims in published maps and institutional affiliations.

\section{Supplementary information}

The online version of this article (https://doi.org/10.1038/s41398-017-0035-0) contains supplementary material.

Received: 14 May 2017 Revised: 27 August 2017 Accepted: 7 September 2017

Published online: 08 December 2017

\section{References}

1. WHO. World Health Organisation, WHO Media centre, Obesity and overweight, Fact sheet No. 311 (2016).

2. Debette, S. et al. Midlife vascular risk factor exposure accelerates structural brain aging and cognitive decline. Neurology 77, 461-468 (2011).

3. Bocarsly, M. E. et al. Obesity diminishes synaptic markers, alters microglial morphology, and impairs cognitive function. Proc. Natl Acad. Sci. USA 112, 15731-15736 (2015).

4. Janowitz, D. et al. Association between waist circumference and gray matter volume in 2344 individuals from two adult community-based samples. Neuroimage 122, 149-157 (2015)

5. Kharabian Masouleh, S. et al. Higher body mass index in older adults is associated with lower gray matter volume: implications for memory performance. Neurobiol. Aging 40, 1-10 (2016).

6. Kurth, F. et al. Relationships between gray matter, body mass index, and waist circumference in healthy adults. Hum. Brain Mapp. 34, 1737-1746 (2013).

7. Gustafson, D. Adiposity indices and dementia. Lancet Neurol. 5, 713-720 (2006).

8. Mueller, K. et al. Sex-dependent influences of obesity on cerebral white matter investigated by diffusion-tensor imaging. PLOS ONE 6, e18544 (2011).

9. Habes, M. et al. Advanced brain aging: relationship with epidemiologic and genetic risk factors, and overlap with Alzheimer disease atrophy patterns. Transl. Psychiatry 6, e775 (2016). 
10. Davatzikos, C., Xu, F., An, Y., Fan, Y. \& Resnick, S. M. Longitudinal progression of Alzheimer's-like patterns of atrophy in normal older adults: the SPARE-AD index. Brain 132, 2026-2035 (2009).

11. Da, X. et al. Integration and relative value of biomarkers for prediction of $\mathrm{MC}$ to AD progression: Spatial patterns of brain atrophy, cognitive scores, APOE genotype and CSF biomarkers. Neuroimage Clin. 4, 164-173 (2014).

12. Isgrò MA, Bottoni P \& Scatena R. in Advances in Cancer Biomarkers 125-143 (ed Scatena, R.) Neuron-specific enolase as a biomarker: biochemical and clinical aspects (Springer, Netherlands, 2015) http://link.springer.com/chapter/10.1007/ 978-94-017-7215-0_9.

13. Streitbürger, D.-P. et al. Validating serum S100B and neuron-specific enolase as biomarkers for the human brain - a combined serum, gene expression and MRI study. PLoS ONE 7, e43284 (2012).

14. Mueller, K. et al. Overweight and obesity are associated with neuronal injury in the human cerebellum and hippocampus in young adults: a combined MRI, serum marker and gene expression study. Transl. Psychiatry 2, e200 (2012).

15. Grabe, H. J. et al. Mental and physical distress is modulated by a polymorphism in the 5-HT transporter gene interacting with social stressors and chronic disease burden. Mol. Psychiatry 10, 220-224 (2004).

16. John, P. D. U. et al. Study of Health in Pomerania (SHIP): a health examination survey in an east German region: objectives and design. Soz Präventivmed. 46 186-194 (2001).

17. Völzke, H. et al. Cohort profile: the Study of Health in Pomerania. Int. J. Epidemiol. 40, 294-307 (2011).

18. ATC-Index. Anatomisch-therapeutisch-chemische Klassifikation mit Tagesdosen Amtliche Fassung des ATC-Index mit DDD Angaben für Deutschland (2007).

19. Nauck, M., Winkler, K., März, W. \& Wieland, H. Quantitative determination of high-, low-, and very-low-density lipoproteins and lipoprotein(a) by agarose gel electrophoresis and enzymatic cholesterol staining. Clin. Chem. 41, 1761-1767 (1995)

20. Hegenscheid, $\mathrm{K}$. et al. Potentially relevant incidental findings on research whole-body MRI in the general adult population: frequencies and management. Eur. Radiol. 23, 816-826 (2012).

21. Casmiro, M. et al. Cerebrospinal fluid and serum neuron-specific enolase concentrations in a normal population. Eur. J. Neurol. 12, 369-374 (2005).
22. Hajduková, L. et al. Biomarkers of brain damage: S100B and NSE concentrations in cerebrospinal fluid--a normative study. Biomed. Res. Int. 2015, 379071 (2015).

23. Chaves, M. L. et al. Serum levels of S100B and NSE proteins in Alzheimer's disease patients. J. Neuroinflammation 7, 6 (2010).

24. Olsson, B. et al. CSF and blood biomarkers for the diagnosis of Alzheimer's disease: a systematic review and meta-analysis. Lancet Neurol. 15, 673-684 (2016).

25. Wolf, O. T. \& Kirschbaum, C. Endogenous estradiol and testosterone levels are associated with cognitive performance in older women and men. Horm. Behav. 41, 259-266 (2002)

26. De Boussac, $H$. et al. Enolase is regulated by liver $X$ receptors. Steroids $\mathbf{9 9}$ 266-271 (2015). Part B.

27. Pintzka, C. W. S. \& Håberg, A. K. Perimenopausal hormone therapy is associated with regional sparing of the CA1 subfield: a HUNT MRI study. Neurobiol. Aging 36, 2555-2562 (2015)

28. Guzel, A. et al. Serum neuron-specific enolase as a predictor of short-term outcome and its correlation with Glasgow Coma Scale in traumatic brain injury. Neurosurg. Rev. 31, 439-445 (2008).

29. Morrison, C. D. et al. High fat diet increases hippocampal oxidative stress and cognitive impairment in aged mice: implications for decreased Nrf2 signaling. J. Neurochem. 114, 1581-1589 (2010).

30. Spyridaki, E. C., Avgoustinaki, P. D. \& Margioris, A. N. Obesity, inflammation and cognition. Curr. Opin. Behav. Sci. 9, 169-175 (2016).

31. Volkow, N. D. et al. Inverse association between bmi and prefrontal metabolic activity in healthy adults. Obesity 17, 60-65 (2009).

32. Schmechel, D. E., Brightman, M. W. \& Marangos, P. J. Neurons switch from non-neuronal enolase to neuron-specific enolase during differentiation. Brain Res. 190, 195-214 (1980).

33. Park, H. R. et al. A high-fat diet impairs neurogenesis: Involvement of lipid peroxidation and brain-derived neurotrophic factor. Neurosci. Lett. $\mathbf{4 8 2}$, 235-239 (2010).

34. Molteni, R., Barnard, R. J., Ying, Z., Roberts, C. K. \& Gómez-Pinilla, F. A high-fat, refined sugar diet reduces hippocampal brain-derived neurotrophic factor, neuronal plasticity, and learning. Neuroscience 112, 803-814 (2002).

35. Gustafson, D., Lissner, L., Bengtsson, C., Björkelund, C. \& Skoog, I. A 24-year follow-up of body mass index and cerebral atrophy. Neurology 63, 1876-1881 (2004). 